\title{
Prof. Dr. Kurt Jellinger: an appreciation on the occasion of his anniversary
}

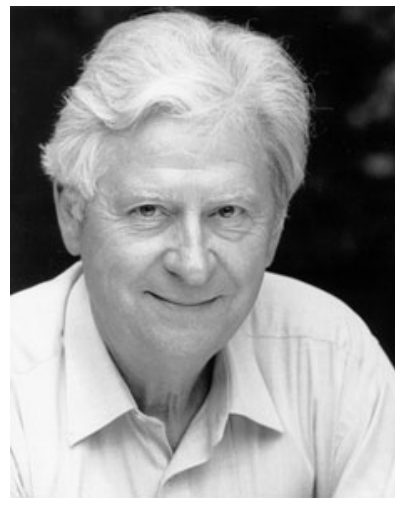

This year, we celebrate the 80th anniversary of Kurt A. Jellinger, Professor of Neurology and Neuropathology at Vienna Medical University, Austria. Kurt Jellinger was staff member of the Neurological Institute at the University of Vienna (1957-1976), chief of the Division of Special Pathology at the Neurological Institute, Vienna, later director of the Department of Neurology, Lainz-Teaching Hospital, Vienna (1976-1997), and director of the Ludwig Boltzmann-Institute of Clinical Neurobiology, Vienna (1977-2002). Since 2002, he is chief of the Institute of Clinical Neurobiology, Vienna, Austria. After decades of a very prominent research career, he is still very active in the field of neuropathology, neuroscience and clinical neurology. The findings of Kurt Jellinger set the stage for subsequent studies for many research groups and his group made very important contributions towards the understanding of the underlying etiology of neurodegenerative diseases including Alzheimer's disease and Parkinson's disease. Kurt authored or co-authored more than 600 scientific publications and his studies and contributions reflect his broad and in-depth interest in a variety of topics relating to neuropathology and brain disease neuropathology. Despite his research focus on neuropathology, Kurt is also a very active clinician and involved in studies to identify biologic markers and to identify symptomatic and biologic therapies to slow the course of neurodegenerative disorders. He combines scientific and clinical work at the highest standard and despite the fact that neuroscience is moving very fast, he was able to keep up his work at highest levels.

During his career, Kurt Jellinger received some of the most prestigious awards in the science community including the Alzheimer Obelisk for research in Alzheimer disease, the Meritorial Service Award of the American Association of Neuropathologists, the Lundbeck Award for Parkinson research, and the A.M. Jakob medal of the German Association of Neuropathology. Kurt Jellinger was executive editor of Acta Neuropathologica (1978-2004) and served on the Editorial Boards of many international scientific journals including JNT. We are all proud and happy to have him on board and look forward to many more years of fruitful and successful cooperation.

Dear Kurt, congratulations for your decades of outstanding research, clinical work, teaching, mentoring and tremendous accomplishments. We have always enjoyed knowing you and interacting with you. We are looking forward to working with you for many more years, especially since your creativity and positive attitude have always been an inspiration for our scientific community.

Josef Marksteiner, Hall, Austria

Peter Riederer, Würzburg, Germany

Würzburg, April, 2011 